Recommendations for gonadotoxicity surveillance in male childhood, adolescent, and young adult cancer survivors : a report from the International Late Effects of Childhood Cancer Guideline Harmonization Group in collaboration with the PanCareSurFup Consortium

\title{
Skinner, Roderick
}

2017-02

Skinner , R , Mulder , R L , Kremer , L C , Hudson , M M , Constine , L S , Bardi , E , Boekhout, A, Borgmann-Staudt, A, Brown, M C , Cohn , R, Dirksen , U , Giwercman , A , Ishiguro , H , Jahnukainen, K, Kenney , L B , Loonen, J J , Meacham , L , Neggers , S , Nussey, S , Petersen , C , Shnorhavorian, M , van den Heuvel-Eibrink, M M , van Santen, H M , Wallace , W H B \& Green , D M 2017, ' Recommendations for gonadotoxicity surveillance in male childhood, adolescent, and young adult cancer survivors : a report from the International Late Effects of Childhood Cancer Guideline Harmonization Group in collaboration with the PanCareSurFup Consortium ' , Lancet Oncology , vol. 18 , no. 2 , pp. E75-E90 . https://doi.org/10.1016/S1470-2045(17)30026-8

http://hdl.handle.net/10138/233868

https://doi.org/10.1016/S1470-2045(17)30026-8

publishedVersion

Downloaded from Helda, University of Helsinki institutional repository.

This is an electronic reprint of the original article.

This reprint may differ from the original in pagination and typographic detail.

Please cite the original version. 


\section{Recommendations for gonadotoxicity surveillance in male childhood, adolescent, and young adult cancer survivors: a report from the International Late Effects of Childhood Cancer Guideline Harmonization Group in collaboration with the PanCareSurFup Consortium

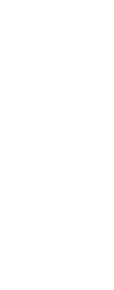

Roderick Skinner, Renee L Mulder, Leontien C Kremer, Melissa M Hudson, Louis S Constine, Edit Bardi, Annelies Boekhout, Anja Borgmann-Staudt,

Morven C Brown, Richard Cohn, Uta Dirksen, Alexsander Giwercman, Hiroyuki Ishiguro, Kirsi Jahnukainen, Lisa B Kenney, Jacqueline J Loonen,

Lilian Meacham, Sebastian Neggers, Stephen Nussey, Cecilia Petersen, Margarett Shnorhavorian, Marry M van den Heuvel-Eibrink,

Hanneke M van Santen, William HB Wallace, Daniel M Green

Treatment with chemotherapy, radiotherapy, or surgery that involves reproductive organs can cause impaired spermatogenesis, testosterone deficiency, and physical sexual dysfunction in male pubertal, adolescent, and young adult cancer survivors. Guidelines for surveillance and management of potential adverse effects could improve cancer survivors' health and quality of life. Surveillance recommendations vary considerably, causing uncertainty about optimum screening practices. This clinical practice guideline recommended by the International Late Effects of Childhood Cancer Guideline Harmonization Group in collaboration with the PanCareSurFup Consortium, developed using evidence-based methodology, critically synthesises surveillance recommendations for gonadotoxicity in male childhood, adolescent, and young adult (CAYA) cancer survivors. The recommendations were developed by an international multidisciplinary panel including 25 experts in relevant medical specialties, using a consistent and transparent process. Recommendations were graded according to the strength of underlying evidence and potential benefit gained by early detection and appropriate management. The aim of the recommendations is to enhance evidence-based care for male CAYA cancer survivors. The guidelines reveal the paucity of high-quality evidence, highlighting the need for further targeted research.

\section{Introduction}

Advances in the treatment of childhood, adolescent, and young adult (CAYA) cancer have led to 5-year survival exceeding $80 \% .^{1,2}$ Unfortunately, many CAYA cancer survivors experience long-term morbidity and mortality resulting from cancer, treatment, or both. ${ }^{3,4}$ The risk of treatment-induced gonadotoxicity often causes emotional distress for male survivors ${ }^{5-10}$ and impaired spermatogenesis results in reduced or lost fertility. ${ }^{5,7}$ Testosterone deficiency causes delayed or arrested puberty in adolescents, and many adverse somatic and psychological consequences in adults. ${ }^{6,8,11,12}$ Sexual dysfunction can have both physical and psychosexual consequences. ${ }^{10,13}$

Male survivors at risk of cancer and treatment-related gonadotoxic effects and their health-care providers could benefit from clinical practice guidelines to facilitate timely remedial or health-preserving interventions. ${ }^{14-17}$ Published clinical practice guidelines developed by North American and European groups for long-term follow-up of CAYA cancer survivors ${ }^{18-21}$ vary in definitions of at-risk populations and recommendations for treatment modalities and frequency of surveillance. Because differences in published guidelines might hinder guideline implementation and provision of consistent clinically effective care, the International Late Effects of Childhood Cancer Guideline Harmonization Group (IGHG) was developed to critically examine the evidence and harmonise existing long-term follow-up guidelines. ${ }^{22}$ The European Union-funded PanCareSurFup (PCSF) Consortium collaborated with IGHG to identify treatments associated with increased risk of impaired spermatogenesis, testosterone deficiency, and physical sexual dysfunction in male CAYA cancer survivors, and evaluate surveillance strategies. This Review summarises the evidence and recommendations. Treatment of impaired spermatogenesis, testosterone deficiency and physical sexual dysfunction by relevant specialist clinicians was not included in this Review.

\section{Aim of the study}

IGHG's aims and methodology have been published. ${ }^{22}$ Guideline representatives from the North American Children's Oncology Group (COG), ${ }^{19}$ Dutch Childhood Oncology Group (DCOG), Scottish Intercollegiate Guidelines Network (SIGN), ${ }^{20}$ United Kingdom Children's Cancer and Leukaemia Group (CCLG), ${ }^{18}$ PCSF Consortium, ${ }^{23}$ and other international paediatric oncology societies developed a working group of 25 experts from nine countries, selected for their knowledge of and publications in paediatric (four members) or adult endocrinology (two members), andrology (one member), paediatric, adolescent haematology, oncology, or long-term follow-up (twelve members), urology (one member), radiation oncology (one member), psychology (one member), and epidemiology and

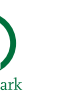

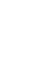


Australia (Prof R Cohn FRACP); Department of Paediatric Haematology and Oncology, University Hospital Muenster, Muenster, Germany (Prof U Dirksen MD); Department of Translational Medicine, Lund University, Malmö, Sweden (Prof A Giwercman MD); Department of Pediatrics, Tokai University School of Medicine, Isehara, Japan (H Ishiguro MD); Division of

Hematology-Oncology and Stem Cell Transplantation, Children's Hospital, University of Helsinki and Helsinki University Hospital, Helsinki, Finland (KJahnukainen PhD); Department of Pediatric Oncology, DanaFarber/Boston Children's Cancer and Blood Disorder Center, Harvard Medical School, Boston, MA, USA (Prof L B Kenney MD); Department of Hematology, Radboud University Medical Center, Nijmegen, Netherlands (J Loonen PhD); Department of Pediatrics, Division of Hematology/Oncology and Endocrinology, Emory University and Aflac Cancer Center of Children's Healthcare of Atlanta, Atlanta, GA, USA (Prof L Meacham MD);

Department of Medicine section Endocrinology, Erasmus University Medical Center Rotterdam and Pediatric Oncology, Sophia's Children's Hospital/Erasmus University Medical Center, Rotterdam, Netherlands (S Neggers PhD); Department of Endocrinology, St George's University of London, London, UK (Prof S Nussey DPhil); Department of Pediatric Oncology, Karolinska University Hospital, Stockholm, Sweden (C Peterson PhD); Department of Urology, University of Washington, Division of

Pediatric Urology, Seattle Children's Hospital, Seattle, WA, USA (M Shnorhavorian MD); Princess Maxima Center for Pediatric Oncology, Utrecht, Netherlands

(Prof M M van den Heuvel-Eibrink MD); Department of Pediatric

Endocrinology, Wilhelmina

Kinderziekenhuis, Universitair

Medisch Centrum Utrecht, Utrecht, Netherlands

( $\mathrm{H}$ M van Santen MD); and

University of Edinburgh and

Royal Hospital for Sick Children, Edinburgh, UK

(ProfWHBWallace MD) guideline methodology (three members). The group worked together via web conferences, face-to-face meetings, and email correspondence.

The experts looked for areas of concordance and discordance across the COG, DCOG, SIGN, and CCLG guidelines. They devised clinical questions to address areas of discordance for surveillance of impaired spermatogenesis, testosterone deficiency, and physical limitations that lead to sexual dysfunction (subsequently described as physical sexual dysfunction) covering the following key issues: who needs surveillance; which surveillance modality should be used; how often and for how long surveillance should be performed; and when survivors should be referred (appendix pp 1-3).

\section{Methods}

We did systematic searches in the medical literature for studies of CAYA survivors (see appendix, pp 4-10, for more details).

\section{Search strategy and selection criteria}

Systematic literature searches were performed between January, 1993 and October, 2014 (appendix, pp 4-10). We searched MEDLINE (through PubMed) using the search terms "childhood cancer", "male", "alkylating agents", "platinum agents", "radiotherapy", "orchidectomy", "impaired spermatogenesis", "testosterone deficiency", "sexual dysfunction", "FSH", "inhibin B", "LH", "testosterone" (detailed search strategies are provided in the appendix, pp 4-10). We critiqued references supporting the existing recommendations and contacted experts in the specialty to determine if any additional evidence was available. Only reports published in English were reviewed. The inclusion criteria were based on study population, outcomes, type, and date of study. Eligible study populations were male human childhood, adolescent, and young adult cancer survivors, in which $75 \%$ or more had been diagnosed with cancer before the age of 25 years and $50 \%$ or more had been followed up for 2 years or more after cancer diagnosis. Eligible study outcomes were impaired spermatogenesis (azoospermia oligozospermia) or testosterone deficiency. All study designs were eligible (including diagnostic studies to address which surveillance modality should be used) if the sample size included 20 patients or more and if the study controlled for important confounding factors, such as treatment or age (eg, cohort study using a multivariable or multiple regression analysis or case control study that used measures to control for important factors). Studies that used only a univariable analysis were excluded. Studies published from 1993 onwards were eligible.

Studies meeting the inclusion criteria were selected and evidence summaries were generated to answer the clinical questions. The final selection of studies was based on relevance to the broad scope of this guideline. In the case of concordance, the evidence cited by the guidelines was evaluated.
Papers were selected for evidence summaries if they reported a primary outcome. Conclusions based on gonadotrophins alone were not used to inform surveillance recommendations for impaired spermatogenesis or testosterone deficiency because they were not defined as primary outcomes and patients treated with cranial radiotherapy might develop hypogonadotropic hypogonadism, and hence blunted gonadotrophin responses.

Studies of surveillance modalities for impaired spermatogenesis calculated the area under the curve (AUC) of the receiver operating characteristics (ROC) curves to evaluate accuracy of a diagnostic investigation. We used Tape's criteria, ${ }^{24}$ based on the numerical value of the AUC of the ROC curve, to analyse the diagnostic value of investigations.

In cases whereby no or only low-quality evidence was identified for the gonadotoxic effect (or absence) of a treatment, relevant information was extrapolated from studies that did not meet the systematic search's eligibility criteria, including studies published before 1993. Conclusions from this supplemental search were agreed upon and described as reflecting expert opinion. They were categorised as probably demonstrating an effect or no effect of the treatment, or as suggesting that the risk was unclear. For a full flowcharton the studies selected see figure 1 .

\section{Definitions used}

Given the heterogeneity in definitions of relevant therapeutic exposures, surveillance strategies and clinical outcomes, we developed standardised definitions for our literature review and final recommendations.

CAYA cancer survivors were defined as individuals diagnosed with cancer up to 25 years of age and at least 2 years after completion of treatment, regardless of current age.

Chemotherapy drugs included in the literature searches were alkylating and similar DNA interstrand crosslinking agents, including bifunctional classical alkylators (busulfan, chlorambucil, cyclophosphamide, ifosfamide, chlormethine, melphalan, thiotepa), platinum agents (carboplatin, cisplatin), and monofunctional nitrosoureas (carmustine, lomustine), and triazenes (dacarbazine, procarbazine, temozolomide). These drugs are subsequently referred to collectively as alkylating agents. Cytarabine was also included in view of reports of potential gonadotoxicity. 25,26

Radiotherapy potentially exposing the testes or pelvis included the following treatment volumes: testes, flank or hemi-abdomen if the radiotherapy field extended below the iliac crest, whole abdomen, inverted Y (paraaortic, iliac, inguinal, and upper femoral lymph nodes, often with spleen or splenic pedicle), pelvis, prostate, bladder, iliac, inguinal, femoral, thigh, total lymphoid irradiation, total nodal irradiation, and total body irradiation (TBI). Although radiotherapy (eg, cranial) that potentially exposes the hypothalamic-pituitary region 
might cause central hypogonadism and was included in our search strategy, we did not develop surveillance recommendations because this will be evaluated in a future IGHG/PCSF pituitary dysfunction guideline.

Primary and other outcomes were defined. For primary outcomes, impaired spermatogenesis was defined as azoospermia or oligozoospermia demonstrated by semen analysis, testosterone deficiency as decreased serum testosterone concentrations, and physical sexual dysfunction as erectile or ejaculatory dysfunction, or impotence. Reference ranges were the same as those used in the selected articles. Other outcomes evaluated included elevated serum follicle-stimulating hormone (FSH), or reduced serum inhibin B concentration (for impaired spermatogenesis), and elevated serum luteinising hormone (LH) concentration (for testosterone deficiency). Delayed puberty was defined as failure to develop signs of puberty by 14 years of age. Failure of pubertal progression was defined as no change in pubertal stage for at least 6 months at the age at which progression would be expected.

\section{Final recommendations}

Final recommendations were based on scientific knowledge in the evidence summaries, combined with other considerations including clinical judgements, decisions about thresholds, costs, potential harms from excessive screening, and the need to maintain flexibility of application across different health-care systems. The quality of the evidence and the strength of the recommendations were graded according to published evidence-based methods (appendix pp 11-12).22,27,28 The harmonised male gonadotoxicity surveillance recommendations were critically appraised by two independent external experts in the field and five survivor representatives.

\section{Findings}

Tables 1-3 show the concordances and discordances between existing surveillance recommendations for impaired spermatogenesis, testosterone deficiency, and physical sexual dysfunction.

Many areas of discordance were identified that required more detailed investigation of the evidence. The appendix presents the clinical questions (pp 1-3), evidence summaries (pp 13-34), and conclusions of evidence tables (pp 35-48) for the evidence summaries, and summarises evidence from and provides references for studies identified by the supplemental search (pp 49-53).

Of 3202 studies of impaired spermatogenesis, testosterone deficiency, and physical sexual dysfunction identified, seven met all inclusion criteria to address who needs surveillance and at what frequency (figure 1A). Of 1170 studies of impaired spermatogenesis and testosterone deficiency identified, six met all inclusion criteria to address which surveillance modality should be used (figure 1B).

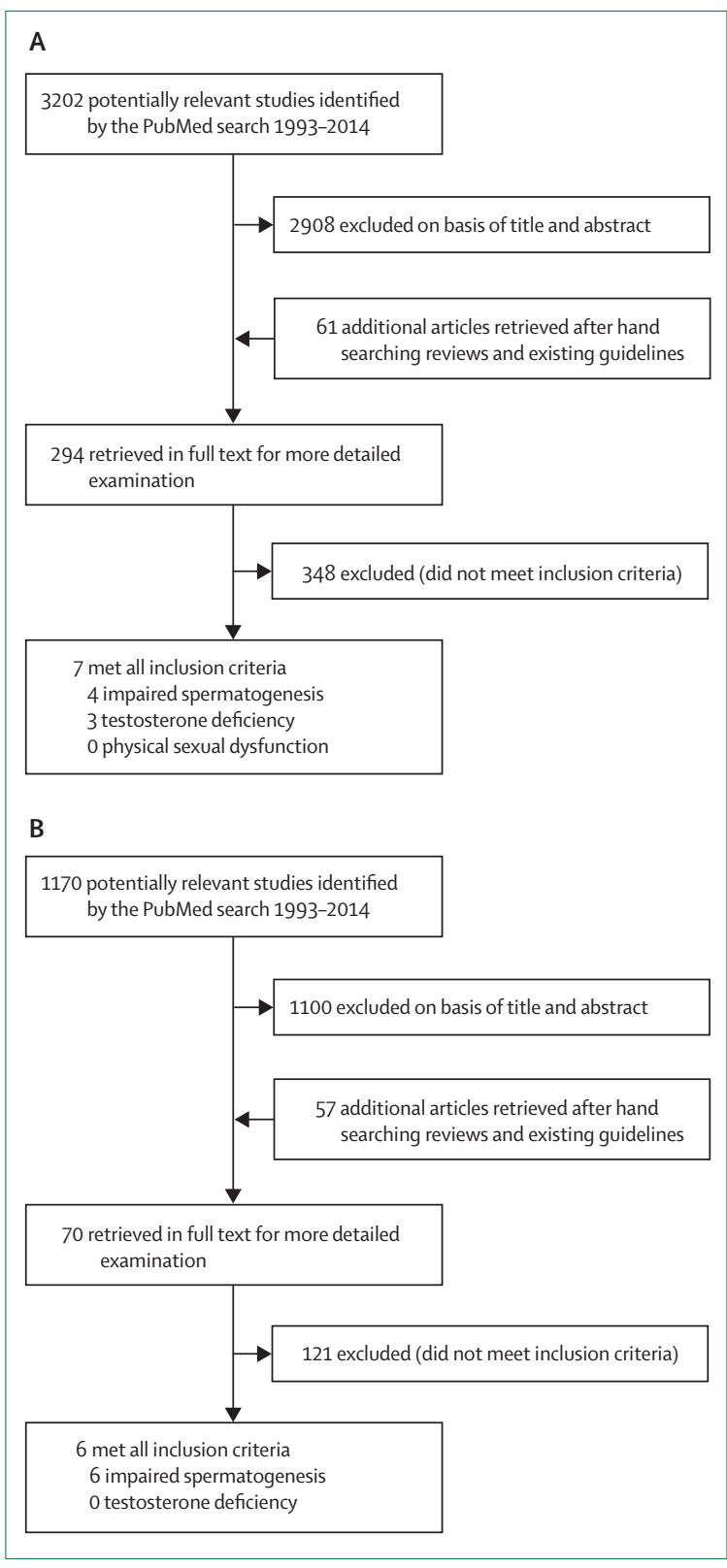

Correspondence to: Prof R Skinner, Department of Paediatric and Adolescent Haematology/Oncology and Children's Haemopoietic Stem Cell Transplant Unit, Great North Children's Hospital, Newcastle upon Tyne NE1 4LP, UK roderick.skinner@ncl.ac.uk

For more on the Dutch Childhood Oncology Group see http://www.skion.nl/

See Online for appendix

For more on Cochrane Childhood Cancer see http://ccg. cochrane.org 


\begin{tabular}{|c|c|c|c|c|c|}
\hline & $\begin{array}{l}\text { North American Children's } \\
\text { Oncology Group }\end{array}$ & $\begin{array}{l}\text { Dutch Childhood } \\
\text { Oncology Group }\end{array}$ & $\begin{array}{l}\text { UK Children's } \\
\text { Cancer and } \\
\text { Leukaemia Group }\end{array}$ & $\begin{array}{l}\text { Scottish } \\
\text { Intercollegiate } \\
\text { Guidelines Network }\end{array}$ & $\begin{array}{l}\text { Concordant } \\
\text { or discordant }\end{array}$ \\
\hline \multicolumn{6}{|c|}{ Who needs impaired spermatogenesis surveillance? } \\
\hline At risk & & All survivors & & & \\
\hline Alkylating agents* & Yes & Not specified & Yes & Yes & Discordant \\
\hline Procarbazine & Yes & Not specified & Yes & Not specified & Discordant \\
\hline Temozolamide, dacarbazine & Yes & Not specified & Yes & Not specified & Discordant \\
\hline Carboplatin, cisplatin & Yes & Not specified & Yes & Not specified & Discordant \\
\hline Cytarabine & Not specified & Not specified & Yes & Not specified & Discordant \\
\hline Radiotherapy exposing testes $\dagger$ & Yes & Not specified & Yes & Yes & Discordant \\
\hline Unilateral orchiectomy & Yes & Not specified & Not specified & Not specified & Discordant \\
\hline \multicolumn{6}{|l|}{ Highest risk } \\
\hline Higher doses alkylating agents & Yes & Not specified & Not specified & Not specified & Discordant \\
\hline Alkylating agent dose & $\begin{array}{l}\text { MOPP } 3 \text { cycles or more } \\
\text { Busulfan }\left(\geq 600 \mathrm{mg} / \mathrm{m}^{2}\right) \\
\text { Cyclophosphamide }\left(\geq 7 \cdot 5 \mathrm{~g} / \mathrm{m}^{2}\right) \\
\text { Cyclophosphamide for HSCT } \\
\text { Ifosfamide }\left(\geq 60 \mathrm{~g} / \mathrm{m}^{2}\right)\end{array}$ & Not specified & Not specified & Not specified & Discordant \\
\hline Combination alkylating agents & Yes & Not specified & Not specified & Not specified & Discordant \\
\hline Higher dose radiotherapy exposing testes & Yes & Not specified & Not specified & Not specified & Discordant \\
\hline Radiotherapy dose & $\begin{array}{l}\text { 1-3 Gy: azoospermia possibly } \\
\text { reversible } \\
\text { 3-6 Gy: azoospermia possibly } \\
\text { reversible (but unlikely) } \\
6 \text { Gy or more: azoospermia } \\
\text { probably permanent }\end{array}$ & Not specified & Not specified & Not specified & Discordant \\
\hline $\begin{array}{l}\text { Alkylating agents and radiotherapy } \\
\text { exposing testes }\end{array}$ & Yes & Not specified & Not specified & Not specified & Discordant \\
\hline $\begin{array}{l}\text { Unilateral orchiectomy and radiotherapy } \\
\text { exposing testes or alkylating agents }\end{array}$ & Yes & Not specified & Not specified & Not specified & Discordant \\
\hline Bilateral orchiectomy $\ddagger$ & Yes & Not specified & Not specified & Not specified & Discordant \\
\hline \multicolumn{6}{|l|}{ Which surveillance modality should be used? } \\
\hline Tanner staging & Yes & Yes & Yes & Yes & Concordant \\
\hline Testicular volume & Yes & Yes & Yes & Yes & Concordant \\
\hline FSH & Yes & Yes & Yes & Yes & Concordant \\
\hline Inhibin B & Not specified & Not specified & Yes & Not specified & Discordant \\
\hline Semen analysis & Yes & Yes & Yes & Yes & Concordant \\
\hline \multicolumn{6}{|c|}{ At what frequency should impaired spermatogenesis surveillance be performed? } \\
\hline Tanner staging & $\begin{array}{l}\text { Every } 1 \text { year (until sexually } \\
\text { mature) }\end{array}$ & Every visit & $\begin{array}{l}\text { Every } 0.5 \text { year } \\
\text { (all survivors, until } \\
\text { sexually mature) }\end{array}$ & Not specified & Discordant \\
\hline Testicular volume & Every 1 year & Every visit & $\begin{array}{l}\text { Every } 0.5 \text { year } \\
\text { (all survivors) }\end{array}$ & Not specified & Discordant \\
\hline FSH & $\begin{array}{l}\text { In sexually mature patients if } \\
\text { unable to obtain semen analysis }\end{array}$ & $\begin{array}{l}\text { As clinically } \\
\text { indicated }\end{array}$ & Every $0.5-1$ years & Not specified & Discordant \\
\hline Inhibin B & N/A & N/A & $\begin{array}{l}\text { Every } 0 \cdot 5-1 \text { years } \\
\text { (if available) }\end{array}$ & $\mathrm{N} / \mathrm{A}$ & $\mathrm{N} / \mathrm{A}$ \\
\hline Semen analysis & As requested by patient & $\begin{array}{l}\text { As clinically } \\
\text { indicated }\end{array}$ & When appropriate & Not specified & $\begin{array}{l}\text { Partly } \\
\text { concordant }\end{array}$ \\
\hline \multicolumn{6}{|c|}{ What should be done when abnormalities are identified? } \\
\hline Refer to specialist & Yes & Yes & Yes & Yes & Concordant \\
\hline Consider assisted reproductive technology & Yes (by consultation) & Not specified & Yes & Yes & Discordant \\
\hline $\begin{array}{l}\text { MOPP=mustargen (chlormethine, nitrogen must } \\
\text { hormone. *Busulfan, chlorambucil, cyclophospha } \\
\text { exposing the testes: whole abdomen, pelvic, testi } \\
\text { femoral lymph nodes, often with spleen or spleni } \\
\text { refer to endocrinology at } 11 \text { years of age for initia }\end{array}$ & $\begin{array}{l}\text { ard), oncovin (vincristine), procarbaz } \\
\text { mide, ifosfamide, chlormethine (nitr } \\
\text { cular, prostate, flank or hemiabdome } \\
\text { c pedicle), bladder, iliac, inguinal, fem } \\
\text { tion of hormonal replacement therap }\end{array}$ & $\begin{array}{l}\text { ne, prednisone. HSCT= } \\
\text { ogen mustard), melph } \\
\text { n (if extended below ili } \\
\text { oral, total lymphoid irr } \\
\text { y to induce puberty. }\end{array}$ & $\begin{array}{l}\text { =haematopoietic ste } \\
\text { halan, thiotepa, carmı } \\
\text { liac crest), inverted Y } \\
\text { radiation, total body }\end{array}$ & $\begin{array}{l}\text { n cell transplant. FSH=fo } \\
\text { stine, lomustine. †Radio } \\
\text { para-aortic, iliac, inguin } \\
\text { irradiation. } \neq \text { Not for scre }\end{array}$ & $\begin{array}{l}\text { licle-stimulating } \\
\text { cherapy potentialll, } \\
\text { l, and upper } \\
\text { ening purposes; }\end{array}$ \\
\hline
\end{tabular}




\begin{tabular}{|c|c|c|c|c|c|}
\hline & $\begin{array}{l}\text { North American Children's } \\
\text { Oncology Group }\end{array}$ & $\begin{array}{l}\text { Dutch Childhood } \\
\text { Oncology Group }\end{array}$ & $\begin{array}{l}\text { UK Children's Cancer } \\
\text { and Leukaemia Group }\end{array}$ & $\begin{array}{l}\text { Scottish Intercollegiate } \\
\text { Guidelines Network }\end{array}$ & $\begin{array}{l}\text { Concordant } \\
\text { or discordant }\end{array}$ \\
\hline \multicolumn{6}{|c|}{ Who needs testosterone deficiency surveillance? } \\
\hline At risk & & All survivors & & & \\
\hline Alkylating agents* & Yes & Not specified & Yes & Yes & Discordant \\
\hline Procarbazine & Yes & Not specified & Yes & Not specified & Discordant \\
\hline Temozolamide, dacarbazine & Yes & Not specified & Yes & Not specified & Discordant \\
\hline Carboplatin, cisplatin & Yes & Not specified & Yes & Not specified & Discordant \\
\hline Cytarabine & Not specified & Not specified & Yes & Not specified & Discordant \\
\hline Radiotherapy exposing testes $\dagger$ & Yes if 20 Gy or more & Not specified & Yes & Yes & Discordant \\
\hline Unilateral orchiectomy & Yes & Not specified & Not specified & Not specified & Discordant \\
\hline \multicolumn{6}{|l|}{ Highest risk } \\
\hline Higher doses alkylating agents & Yes & Not specified & Not specified & Not specified & Discordant \\
\hline Alkylating agent dose & $\begin{array}{l}\text { MOPP } \\
\text { Cyclophosphamide }\left(\geq 20 \mathrm{~g} / \mathrm{m}^{2}\right) \\
\text { Cyclophosphamide for HSCT } \\
\text { Ifosfamide }\left(\geq 60 \mathrm{~g} / \mathrm{m}^{2}\right)\end{array}$ & Not specified & Not specified & Not specified & Discordant \\
\hline Combination alkylating agents & Yes & Not specified & Not specified & Not specified & Discordant \\
\hline $\begin{array}{l}\text { Higher doses radiotherapy } \\
\text { exposing testes }\end{array}$ & Yes & Not specified & Not specified & Not specified & Discordant \\
\hline Radiotherapy dose & 20 Gy or more & Not specified & Not specified & Not specified & Discordant \\
\hline Radiotherapy exposing testis & Yes & Not specified & Not specified & Not specified & Discordant \\
\hline $\begin{array}{l}\text { Alkylating agents and } \\
\text { radiotherapy exposing testes }\end{array}$ & Yes & Not specified & Not specified & Not specified & Discordant \\
\hline Bilateral orchiectomy $\ddagger$ & Yes & Not specified & Not specified & Not specified & Discordant \\
\hline \multicolumn{6}{|c|}{ Which surveillance modality should be used? } \\
\hline Height and weight & Not specified & Yes & Yes & Yes & Discordant \\
\hline Testicular volume & Yes & Yes & Yes & Yes & Concordant \\
\hline LH & Not specified & Yes & Yes & Yes & Discordant \\
\hline Testosterone & Yes & Yes & Yes & Yes & Concordant \\
\hline \multicolumn{6}{|c|}{ At what frequency should testosterone deficiency surveillance be performed? } \\
\hline Tanner staging & Every 1 year (until sexually mature) & Every visit & $\begin{array}{l}\text { Every } 0.5 \text { year (all } \\
\text { survivors, until } \\
\text { sexually mature) }\end{array}$ & Not specified & Discordant \\
\hline Testicular volume & Every 1 year & Every visit & $\begin{array}{l}\text { Every } 0.5 \text { year (all } \\
\text { survivors) }\end{array}$ & Not specified & Discordant \\
\hline LH & $\mathrm{N} / \mathrm{A}$ & $\begin{array}{l}\text { As clinically } \\
\text { indicated }\end{array}$ & Every $0.5-1$ years & Not specified & Discordant \\
\hline Testosterone & $\begin{array}{l}\text { Baseline at age } 14 \text { years and as } \\
\text { clinically indicated (ideally morning) }\end{array}$ & $\begin{array}{l}\text { As clinically } \\
\text { indicated }\end{array}$ & Every $0.5-1$ years & Not specified & Discordant \\
\hline \multicolumn{6}{|c|}{ What should be done when abnormalities are identified? } \\
\hline Refer to specialist & Yes & Yes & Yes & Yes & Concordant \\
\hline $\begin{array}{l}\text { Consider assisted reproductive } \\
\text { technology }\end{array}$ & Not specified & Not specified & Yes & Yes & Discordant \\
\hline $\begin{array}{l}\text { MOPP=mustargen (chlormethine, nit } \\
\text { *Busulfan, chlorambucil, cyclophosph } \\
\text { abdomen, pelvic, testicular, prostate, } \\
\text { spleen or splenic pedicle), bladder, iliac, } \\
\text { age for initiation of hormonal replace }\end{array}$ & $\begin{array}{l}\text { lank or hemi-abdomen (if extended belo } \\
\text { inguinal, femoral, total lymphoid irradiat } \\
\text { ment therapy to induce puberty. }\end{array}$ & $\begin{array}{l}\text { carbazine, prednison } \\
\text { lan, thiotepa, carmus } \\
\text { wiliac crest), invertec } \\
\text { tion, total body irradi }\end{array}$ & 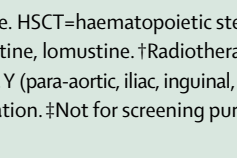 & $\begin{array}{l}\text { apy potentially exposing th } \\
\text { and upper femoral lymph n } \\
\text { rposes; refer to endocrinolc }\end{array}$ & $\begin{array}{l}\text { nising hormone. } \\
\text { testes: whole } \\
\text { des, often with } \\
\text { gy at 11 years of }\end{array}$ \\
\hline
\end{tabular}

spermatogenesis (level $\mathrm{C}$ evidence). ${ }^{29,30}$ Higher doses had greater risks than did lower doses (levels B and C). ${ }^{29-31}$ However, one study ${ }^{30}$ suggested that higher dacarbazine doses were no more toxic to spermatogenesis than lower doses (level C) but no studies compared patients who received or did not receive dacarbazine. No studies evaluated impaired spermatogenesis risk after treatment with other alkylating agents, platinum agents, or cytarabine.

Only weak evidence from the systematic search showed that TBI was associated with increased impaired spermatogenesis risk (level C). ${ }^{32}$ No studies compared 


\begin{tabular}{|c|c|c|c|c|c|}
\hline & $\begin{array}{l}\text { North American Children's } \\
\text { Oncology Group }\end{array}$ & $\begin{array}{l}\text { Dutch Childhood } \\
\text { Oncology Group }\end{array}$ & $\begin{array}{l}\text { UK Children's Cancer } \\
\text { and Leukaemia Group }\end{array}$ & $\begin{array}{l}\text { Scottish Intercollegiate } \\
\text { Guidelines Network }\end{array}$ & $\begin{array}{l}\text { Concordant } \\
\text { or discordant }\end{array}$ \\
\hline \multicolumn{6}{|c|}{ Who needs physical sexual dysfunction surveillance? } \\
\hline \multicolumn{6}{|l|}{ At risk } \\
\hline Neurosurgery spinal cord & Yes & Not specified & Not specified & Not specified & Discordant \\
\hline Pelvic surgery & Yes & Not specified & Not specified & Not specified & Discordant \\
\hline Cystectomy & Yes & Not specified & Not specified & Not specified & Discordant \\
\hline $\begin{array}{l}\text { Radiotherapy exposing bladder, } \\
\text { pelvis, spine }\end{array}$ & Yes & Not specified & Not specified & Not specified & Discordant \\
\hline Hypogonadism & Yes & Not specified & Not specified & Not specified & Discordant \\
\hline Tumour adjacent to spine & Yes & Not specified & Not specified & Not specified & Discordant \\
\hline Radical prostatectomy & Yes & Not specified & Not specified & Not specified & Discordant \\
\hline $\begin{array}{l}\text { Retroperitoneal tumour resection or } \\
\text { node dissection }\end{array}$ & Yes & Not specified & Not specified & Not specified & Discordant \\
\hline \multicolumn{6}{|l|}{ Highest risk } \\
\hline Higher doses radiotherapy & Yes & Not specified & Not specified & Not specified & Discordant \\
\hline Radiotherapy dose & $\begin{array}{l}55 \text { Gy or more to penile } \\
\text { bulb in adults } \\
45 \text { Gy or more in } \\
\text { prepubertal children }\end{array}$ & Not specified & Not specified & Not specified & Discordant \\
\hline Injury above level of sacrum & Yes & Not specified & Not specified & Not specified & Discordant \\
\hline \multicolumn{6}{|c|}{ Which surveillance modality should be used? } \\
\hline $\begin{array}{l}\text { Sexual function (erections, nocturnal } \\
\text { emissions, libido) }\end{array}$ & Yes & Not specified & Not specified & Not specified & Discordant \\
\hline $\begin{array}{l}\text { Medication use impacting sexual } \\
\text { function }\end{array}$ & Yes & Not specified & Not specified & Not specified & Discordant \\
\hline Quality of ejaculate & Yes & Not specified & Not specified & Not specified & Discordant \\
\hline \multicolumn{6}{|c|}{ At what frequency should physical sexual dysfunction surveillance be performed? } \\
\hline Sexual function & Every 1 year & Not specified & Not specified & Not specified & Discordant \\
\hline Medication use & Every 1 year & Not specified & Not specified & Not specified & Discordant \\
\hline Quality of ejaculate & Every 1 year & Not specified & Not specified & Not specified & Discordant \\
\hline \multicolumn{6}{|c|}{ What should be done when abnormalities are identified? } \\
\hline Refer to specialist & Yes & Not specified & Not specified & Not specified & Discordant \\
\hline
\end{tabular}

different testicular radiotherapy doses, or evaluated risk after unilateral orchiectomy.

In the supplemental search, we identified several studies with important limitations (in terms of failing to meet all the inclusion criteria), but still reflecting expert opinion (appendix pp 49-53 for references). It was agreed that an increased risk of impaired spermatogenesis probably exists after treatment with busulfan and cyclophosphamide, or fludarabine and melphalan, haematopoietic stem-cell transplant (HSCT) conditioning, ifosfamide doses of more than $60 \mathrm{~g} / \mathrm{m}^{2}$, radiotherapy potentially exposing testes to more than $2-3 \mathrm{~Gy}$, and TBI. Transient impairment of spermatogenesis is expected and permanent impairment has been reported after radiotherapy treatment potentially exposing testes to less than 2-3 Gy, but there is probably not a substantially increased risk after these small doses, or after unilateral orchiectomy. The risks of impaired spermatogenesis after treatment with cisplatin are unclear.

The panel recommended that survivors treated with one or more potentially gonadotoxic agents should be advised of, and counselled about, the risk of impaired spermatogenesis and its implications for future fertility (strong recommendations; level C evidence, supplemental search and expert opinion). Agents with potential to impair spermatogenesis include cyclophosphamide, chlormethine, and procarbazine (level C), ifosfamide, busulfan/ cyclophosphamide or fludarabine/melphalan, HSCT conditioning, and radiotherapy potentially exposing the testes (supplemental search and expert opinion). Because of interpatient variability and insufficient evidence, it is not possible to make recommendations regarding riskstratified surveillance strategies based on either dose thresholds or age at treatment.

\section{Which surveillance modality should be used?}

The existing surveillance recommendations were concordant in stating that Tanner stage $\mathrm{e}^{33,34}$ and testicular volume, serum FSH, and semen analysis should be evaluated, but discordant for serum inhibin B measurement.

We found three studies ${ }^{7,35,36}$ that investigated the diagnostic value of serum FSH and inhibin B 
concentrations in detecting azoospermia, one study for excluding azoospermia, ${ }^{37}$ and one for detecting oligospermia (defined as $<20 \times 10^{6} / \mathrm{mL}$ ). ${ }^{38}$ One study ${ }^{32}$ evaluated the diagnostic value of serum FSH for excluding azoospermia. Two studies ${ }^{35,36}$ also investigated the diagnostic value of the combination of serum inhibin $\mathrm{B}$ and FSH for detecting azoospermia and one for detecting oligospermia. ${ }^{38}$ The results are summarised in the appendix (pp 43-45). AUCs of the ROC curve are approximately $0 \cdot 7-0 \cdot 8$, suggesting that measurements of $\mathrm{FSH}$, inhibin $\mathrm{B}$, and inhibin $\mathrm{B}$ to $\mathrm{FSH}$ ratio all have fair diagnostic value for detecting azoospermia (level B evidence for FSH and inhibin B, level C for inhibin B to FSH ratio).

The panel recommended that for survivors who desire assessment about possible future fertility after treatment with potentially gonadotoxic therapy, semen analysis should be the gold standard as the primary surveillance modality for evaluating spermatogenesis (strong recommendation; expert opinion).

Clinical measurement of testicular volume, serum FSH, and inhibin B might be reasonable tests for identifying impaired spermatogenesis in survivors treated with potentially gonadotoxic therapy in whom semen analysis has been declined or is not possible, and who desire assessment about their possible future fertility (weak recommendation; level B evidence). However, it is important to be aware of the diagnostic limitations of these tests (appendix pp 25-29, 39-41).

\section{At what frequency and for how long should surveillance be performed?}

The existing surveillance recommendations are discordant regarding the frequency and duration of surveillance for impaired spermatogenesis, except for partial concordance regarding semen analysis.

No studies were identified that provided information about the probability, rate, or timing of changes (improvement or deterioration) in spermatogenesis parameters in male survivors treated with potentially gonadotoxic therapy.

The panel recommended surveillance for impaired spermatogenesis be performed only at the request of the survivor after informed discussion or when paternity is desired in the foreseeable future (strong recommendation; expert opinion).

\section{When should survivors be referred?}

The existing surveillance recommendations are concordant regarding the timing of referral for impaired spermatogenesis but discordant regarding situations in which assisted reproductive technologies should be considered. A literature search was not performed because we considered that expert opinion allowed formulation of a pragmatic referral recommendation.

It is recommended that referral to a male reproductive health specialist should be offered to survivors with severe oligozoospermia (sperm counts $\leq 5 \times 10^{6} / \mathrm{mL}$ ), to individuals seeking paternity after potentially gonadotoxic therapy, or to survivors whose attempts to conceive have been unsuccessful for 6 months or more,$^{39}$ regardless of sperm count, for detailed specialist counselling or consideration of sperm cryopreservation if not already performed (strong recommendation; expert opinion).

\section{Testosterone deficiency}

The clinical presentation of testosterone deficiency varies with the age at onset. In prepubertal and peripubertal males, testosterone deficiency causes delayed or arrested puberty, respectively. In postpubertal males, testosterone deficiency leads to reduced sexual function and numerous systemic consequences including fatigue and increased cardiovascular risk. ${ }^{40}$

Who needs surveillance?

The existing surveillance recommendations were discordant with respect to which chemotherapy, radiotherapy, and surgery exposures place CAYA cancer survivors at risk of testosterone deficiency.

In the systematic search, two studies ${ }^{41,42}$ revealed no increased risk of testosterone deficiency after cyclophosphamide treatment (level C evidence); one study ${ }^{42}$ also found no increased risk after procarbazine or platinum agents (level C). Another study ${ }^{43}$ found no increased risk after higher chlorambucil $\left(672 \mathrm{mg} / \mathrm{m}^{2}\right)$ and procarbazine $\left(11200 \mathrm{mg} / \mathrm{m}^{2}\right)$ doses, compared with lower doses (504 and $8400 \mathrm{mg} / \mathrm{m}^{2}$; level C). No studies compared different cyclophosphamide or platinum agent doses. No studies investigated risk after other alkylating agents or cytarabine.

One study ${ }^{42}$ found no increased testosterone deficiency risk after radiotherapy potentially exposing the testes (pelvic or abdominal, or TBI, doses not specified; level C). No studies were identified that addressed risk after different radiotherapy doses, or after unilateral orchiectomy.

One multivariable study ${ }^{42}$ showed that age at treatment had no effect on testosterone deficiency risk, although only a minority of survivors had received radiotherapy potentially exposing the testes (level C).

In the supplemental search, several studies with important limitations (in terms of failing to meet all inclusion criteria), but reflecting expert opinion, were identified (appendix pp 49-53 for references). An increased risk of testosterone deficiency is probable after radiotherapy treatment that potentially exposes the testes to more than $12 \mathrm{~Gy}$ of radiation or with TBI (7-5-15 Gy). An increased risk of testosterone deficiency is unlikely after radiotherapy potentially exposing testes to less than $12 \mathrm{~Gy}$. According to this evidence and comparability with contemporary TBI schedules, a consensus was reached within the group that a testicular radiotherapy dose threshold of 12 Gy or more, or TBI, is probably associated with increased risk.

An increased risk after treatment with cyclophosphamide, busulfan and cyclophosphamide, or fludarabine 
and melphalan, HSCT conditioning, procarbazine and chlormethine, ifosfamide, and cisplatin is probable.

The panel recommended that survivors treated with a potentially gonadotoxic agent should be advised of, and counselled about, the risk of testosterone deficiency and its implications for future health and fertility (strong recommendations; supplemental search or expert opinion). Treatments with the potential to cause testosterone deficiency include radiotherapy potentially exposing the testes to 12 Gy or more, and TBI (supplemental search or expert opinion). No recommendation can be made regarding risk-stratified surveillance strategies based on age at treatment.

Which surveillance modality should be used, at what frequency, and for how long?

The existing surveillance recommendations for testosterone deficiency are concordant in stating that Tanner stage and testicular volume should be evaluated, and serum testosterone and LH concentration measured. They are partially concordant for measuring height and weight, recommended for all survivors by the CCLG, DCOG, and SIGN guidelines but only for detecting precocious puberty by the COG guidelines. They are discordant regarding the frequency and duration of surveillance.

No studies were identified in the systematic or supplemental searches regarding the diagnostic value of single, compared with serial, serum LH or testosterone measurements to detect testosterone deficiency in postpubertal male survivors treated with potentially gonadotoxic therapy. Similarly, no studies were identified that provided information about the probability, rate, or timing of changes (improvement or deterioration) in testosterone deficiency.

The clinical presentation of testosterone deficiency in CAYA cancer survivors depends on age and can include delayed or arrested puberty, or a variety of clinical manifestations in postpubertal males. The following recommendations describe surveillance for each scenario.

Monitoring of growth (height) and pubertal development and progression (Tanner stage, testicular volume) is recommended for prepubertal and peripubertal cancer survivors treated with radiotherapy potentially exposing testes to $12 \mathrm{~Gy}$ or more, or with TBI (strong recommendation; expert opinion). This monitoring should commence at no later than 12 years of age and should be performed at least annually, with increasing frequency as clinically indicated depending on growth and pubertal progress. In survivors who received cranial radiation, it should be noted that the pubertal increase in growth velocity may be impaired if growth hormone deficiency is also present.

Measurement of total testosterone concentration in an early morning blood sample at clinically appropriate intervals is reasonable in postpubertal survivors treated with radiotherapy potentially exposing the testes to $12 \mathrm{~Gy}$ or more, or with TBI (moderate recommendation; expert opinion). In the presence of clinical signs of hypogonadism, previous low-normal or borderline testosterone concentrations, or in cases whereby it is not possible to obtain an early morning blood sample, measurement of $\mathrm{LH}$ concentration is reasonable in addition to testosterone (moderate recommendation; expert opinion).

\section{When should survivors be referred?}

The existing surveillance recommendations are concordant regarding the timing of referral for testosterone deficiency. A literature search was not performed because we considered that expert opinion allowed formulation of a pragmatic referral recommendation.

Referral to a paediatric endocrinologist is recommended for any male survivor who has no signs of puberty by 14 years of age, or who shows failure of pubertal progression (strong recommendation; expert opinion).

Referral to a specialist in male reproductive health, andrology, endocrinology, or urology (depending on local referral pathways) is recommended for postpubertal male survivors treated with radiotherapy potentially exposing the testes to 12 Gy or more, or with TBI, and in whom laboratory results (reduced serum testosterone or increased serum LH concentration) suggest testosterone deficiency (strong recommendation; expert opinion).

\section{Physical sexual dysfunction \\ Who needs surveillance?}

The existing surveillance recommendations were discordant with respect to which chemotherapy, radiotherapy, and surgery exposures place CAYA cancer survivors at risk of physical sexual dysfunction.

The systematic search identified no studies reporting on risks for treatment-related physical sexual dysfunction after childhood cancer. The supplemental search identified several studies with important limitations (in terms of failing to meet all inclusion criteria), but reflecting expert opinion, were identified (appendix pp 49-53 for references). These studies suggested that hypogonadism (ie, decreased serum testosterone) is probably associated with an increased risk of sexual dysfunction. Surgery to the spinal cord, sympathetic nerves or pelvis, or radiotherapy potentially exposing the testes or pelvis, are probably each associated with an increased risk of sexual dysfunction.

The panel recommended that survivors treated with one or more treatment modalities with the potential to cause physical sexual dysfunction should be advised of, and counselled about, the risk of such dysfunction and its implications for future health and fertility (strong recommendation; supplemental search and expert opinion). Treatments with potential to cause physical sexual dysfunction include surgery to the spinal cord, sympathetic nerves or pelvis, radiotherapy potentially 
exposing the testes or pelvis, or any treatment that causes hypogonadism (supplemental search and expert opinion).

\section{Which surveillance modality should be used?}

The existing surveillance recommendations are discordant regarding surveillance modalities for physical sexual dysfunction. We did not perform a search as clinical history is the only available surveillance tool.

It is recommended that health-care providers should take a relevant sexual history in postpubertal survivors treated with surgery to the spinal cord, sympathetic nerves or pelvis, or radiotherapy potentially exposing the testes or pelvis, or individuals who have been diagnosed with hypogonadism (strong recommendation; expert opinion).

It is not possible to formulate any recommendations about the frequency or duration of surveillance for physical sexual dysfunction in survivors.

\section{When should survivors be referred?}

The existing surveillance recommendations are discordant for the timing of referral for physical sexual dysfunction. A literature search was not performed because we considered that expert opinion allowed formulation of a pragmatic referral recommendation.

The panel recommended referral to a specialist in male reproductive health, andrology, endocrinology, or urology (according to local referral pathways) for survivors treated with surgery to the spinal cord, sympathetic nerves or pelvis, or radiotherapy potentially exposing the testes or pelvis, or those who have hypogonadism, and have symptoms suggestive of physical sexual dysfunction (strong recommendation; expert opinion).

\section{Discussion}

We present the international harmonised surveillance recommendations for gonadal dysfunction in male CAYA cancer survivors. Table 4 summarises the conclusions of the evidence from both the systematic and supplemental searches. Figure 2 presents the final recommendations and the panel presents the gaps in knowledge and future directions for research. The recommendations were derived from critical analysis of the published literature, using strict standards to grade supporting evidence, supplemented by expert consensus in areas in which little or no evidence was available.

Few published studies performing semen analysis or measuring serum testosterone in male CAYA cancer survivors were identified by our systematic search. Additionally, many studies did not mention whether testosterone was measured in early morning samples, limiting their utility. The search provided clear evidence for the gonadotoxicity of some modalities used in the treatment of CAYA cancer, but did not provide evidence for several other modalities that are widely believed to cause impaired spermatogenesis or testosterone deficiency (eg, radiotherapy exposing the testes). Evidence for associations between certain treatment modalities and impaired spermatogenesis or testosterone deficiency derives from studies that did not evaluate the primary outcomes (semen analysis, serum testosterone), were performed in other populations (eg, adults $>25$ years), included less than 20 patients, did not use statistical analyses that accounted for confounding factors, or predated 1993, and hence did not meet the systematic search's inclusion criteria. Although many older publications (pre-1993) provide important insights, most did not evaluate the primary outcomes chosen or account for confounding factors. In view of the importance of the additional evidence that is available from the literature that did not meet the inclusion criteria, we performed a further supplemental search to evaluate this evidence in more detail. For some questions, the supplemental search provided data concerning risks and dose thresholds for toxicity of chemotherapeutic agents and testicular radiotherapy, optimising identification of treatments with or without evidence of probable gonadotoxicity, and assisting formulation of our recommendations. To ensure transparency of the guideline development process, the results of the systematic and supplemental searches are presented separately, and their contributions to the recommendations stated clearly.

The systematic search provided evidence for adverse effects of cyclophosphamide, chlormethine and procarbazine on spermatogenesis, whereas the supplemental search demonstrated evidence for the probable toxicity of busulfan and cyclophosphamide, or fludarabine and melphalan combinations, and ifosfamide. Longstanding clinical experience has shown that testicular radiotherapy impairs spermatogenesis. ${ }^{6,44}$ The supplemental search provided evidence to support this observation from several sources including univariable studies, studies employing outcomes other than semen analysis (eg, FSH), or performed in adult cancer survivors. Although one study ${ }^{32}$ found no increased risk of impaired spermatogenesis after TBI on multivariable regression analysis, the comparator (non-TBI) group included individuals given other potentially gonadotoxic treatments including cyclophosphamide and busulfan. Therefore, for TBI, greater emphasis was placed on the results of the supplemental search. We identified no evidence that age at exposure influences susceptibility to impaired spermatogenesis, thus it appears reasonable to extrapolate evidence from adult studies.

Risk factor evaluation for impaired spermatogenesis, particularly exposure to specific treatment agents, is problematic. Potentially gonadotoxic drugs are frequently used in combination chemotherapy regimens and can also be used with radiotherapy potentially exposing the testes, sometimes with uncertain dosimetry. Many studies are small, retrospective, use varied doses, and do not allow for confounding factors. Alkylating agents used in combination are considered to have an additive adverse effect on spermatogenesis. ${ }^{6}$ Time since treatment 


\begin{tabular}{|c|c|}
\hline & Evidence \\
\hline \multicolumn{2}{|l|}{ Impaired spermatogenesis } \\
\hline Impaired spermatogenesis risk* in CAYA cancer survivors & Evidence from systematic search \\
\hline Increased risk after cyclophosphamide us no cyclophosphamide & Level C ${ }^{29}$ \\
\hline Increased risk after higher cyclophosphamide dose vs lower dose & Level B $\mathrm{B}^{29,31}$ \\
\hline Increased risk after chlormethine vs no chlormethine $\dagger$ & Level $3^{30}$ \\
\hline Increased risk after higher chlormethine dose vs lower dose & Level C ${ }^{30}$ \\
\hline Increased risk after procarbazine vs no procarbazine $\neq$ & Level $3^{30}$ \\
\hline Increased risk after higher procarbazine dose vs lower dose & Level $3^{30}$ \\
\hline Risk after dacarbazine vs no dacarbazine & No studies \\
\hline No increased risk after higher dacarbazine dose vs lower dose & Level ${ }^{30}$ \\
\hline Risk after temozolomide & No studies \\
\hline Risk after other alkylating agents & No studies \\
\hline Risk after platinating agents $\mathbf{I}$ & No studies \\
\hline Risk after cytarabine & No studies \\
\hline No increased risk after radiotherapy potentially exposing the testes given as TBI vs no radiotherapy & Level $\mathrm{C}^{32}$ \\
\hline Risk after higher dose of radiotherapy potentially exposing the testes vs lower dose & No studies \\
\hline Risk after unilateral orchiectomy & No studies \\
\hline Impaired spermatogenesis risk* in cancer survivors & Evidence from supplemental search \\
\hline Increased risk probable after busulfan and cyclophosphamide for HSCT & Expert opinion\| \\
\hline Increased risk probable after fludarabine and melphalan for HSCT & Expert opinion\| \\
\hline Increased risk probable after ifosfamide $\left(>60 \mathrm{~g} / \mathrm{m}^{2}\right)$ & Expert opinion\| \\
\hline Unclear risk after cisplatin & Expert opinion\| \\
\hline Probably no increased risk after radiotherapy potentially exposing the testes to less than 2-3 Gy & Expert opinion|| \\
\hline Increased risk probable after radiotherapy potentially exposing the testes to more than 2-3 Gy & Expert opinion\| \\
\hline Increased risk probable after radiotherapy potentially exposing the testes given as TBI & Expert opinion\| \\
\hline Probably no increased risk after unilateral orchiectomy & Expert opinion\| \\
\hline Diagnostic value endocrine measurement to detect impaired spermatogenesis in CAYA cancer survivors & Evidence from systematic search \\
\hline Fair diagnostic value of inhibin B to detect azoospermia & Level B $B^{7,35-37}$ \\
\hline Fair diagnostic value of FSH to detect azoospermia & Level $B^{7,32,35-37}$ \\
\hline Fair diagnostic value of inhibin B to FSH ratio to detect azoospermia & Level $C^{35}$ \\
\hline Impaired spermatogenesis risk in CAYA cancer survivors & $\begin{array}{l}\text { Evidence from systematic and supplemental } \\
\text { searches }\end{array}$ \\
\hline Likelihood or timing of changes (deterioration or improvement) in spermatogenesis parameters & No studies \\
\hline \multicolumn{2}{|l|}{ Testosterone deficiency } \\
\hline Testosterone deficiency risk in CAYA cancer survivors & Evidence from systematic search \\
\hline No increased risk after cyclophosphamide vs no cyclophosphamide & Level Ci,42 \\
\hline Risk after higher cyclophosphamide dose vs lower dose & No studies \\
\hline Risk after chlorambucil vs no chlorambucil & No studies \\
\hline No increased risk after higher chlorambucil dose vs lower dose ${ }^{\dagger}$ & Level $C^{43}$ \\
\hline No increased risk after procarbazine vs no procarbazine & Level $C^{42}$ \\
\hline No increased risk after higher procarbazine dose vs lower dose ${ }^{* *}$ & Level ${ }^{43}$ \\
\hline Risk after dacarbazine & No studies \\
\hline Risk after temozolomide & No studies \\
\hline Risk after other alkylating agents $\dagger \dagger$ & No studies \\
\hline No increased risk after platinating agents $\mathbf{I}$ vs no platinating agents & Level C ${ }^{43}$ \\
\hline Risk after higher platinating agents $\boldsymbol{I}$ dose vs lower dose & No studies \\
\hline Risk after cytarabine & No studies \\
\hline $\begin{array}{l}\text { No increased risk after radiotherapy potentially exposing the testes given as TBI or pelvic or abdominal } \\
\text { radiotherapy vs no radiotherapy }\end{array}$ & Level ${ }^{43}$ \\
\hline Risk after higher dose of radiotherapy potentially exposing the testes vs lower dose & No studies \\
\hline \multirow[t]{2}{*}{ Risk after unilateral orchiectomy } & No studies \\
\hline & (Table 4 continues on next page) \\
\hline
\end{tabular}




\begin{tabular}{|c|c|}
\hline & Evidence \\
\hline \multicolumn{2}{|l|}{ (Continued from previous page) } \\
\hline Testosterone deficiency risk in cancer survivors & Evidence from supplemental searches \\
\hline Probably no increased risk after cyclophosphamide & Expert opinion\| \\
\hline Probably no increased risk after busulfan and cyclophosphamide for HSCT & Expert opinion\| \\
\hline Probably no increased risk after fludarabine and melphalan for HSCT & Expert opinion\| \\
\hline Probably no increased risk after procarbazine and chlormethine & Expert opinion\| \\
\hline Probably no increased risk after ifosfamide & Expert opinion\| \\
\hline Probably no increased risk after cisplatin & Expert opinion\| \\
\hline Probably no increased risk after radiotherapy potentially exposing the testes to less than $12 \mathrm{~Gy}$ & Expert opinion\| \\
\hline Probably increased risk after radiotherapy potentially exposing the testes to $12 \mathrm{~Gy}$ or more & Expert opinion\| \\
\hline Probably increased risk after radiotherapy potentially exposing the testes given as TBI & Expert opinion\| \\
\hline Diagnostic value endocrine measurement to detect testosterone deficiency in CAYA cancer survivors & $\begin{array}{l}\text { Evidence from systematic and supplemental } \\
\text { searches }\end{array}$ \\
\hline Diagnostic value of luteinising hormone to detect testosterone deficiency & No studies \\
\hline Testosterone deficiency risk in CAYA cancer survivors & $\begin{array}{l}\text { Evidence from systematic and supplemental } \\
\text { searches }\end{array}$ \\
\hline Likelihood or timing of changes (deterioration or improvement) of testosterone levels & No studies \\
\hline \multicolumn{2}{|l|}{ Physical sexual dysfunction } \\
\hline Physical sexual dysfunction risk in CAYA cancer survivors & Evidence from systematic search \\
\hline Risk after surgery to the spinal cord, sympathetic nerves, or pelvis & No studies \\
\hline Risk after radiotherapy potentially exposing the testes or pelvis & No studies \\
\hline Risk in patients who are hypogonadal (decreased testosterone) & No studies \\
\hline Physical sexual dysfunction risk in cancer survivors & Evidence from supplemental searches \\
\hline Probably increased risk after surgery to the spinal cord, sympathetic nerves, or pelvis & Expert opinion\| \\
\hline Probably increased risk after radiotherapy potentially exposing the testes or pelvis & Expert opinion\| \\
\hline Probably increased risk in patients who are hypogonadal (decreased testosterone) & Expert opinion\| \\
\hline \multicolumn{2}{|c|}{ 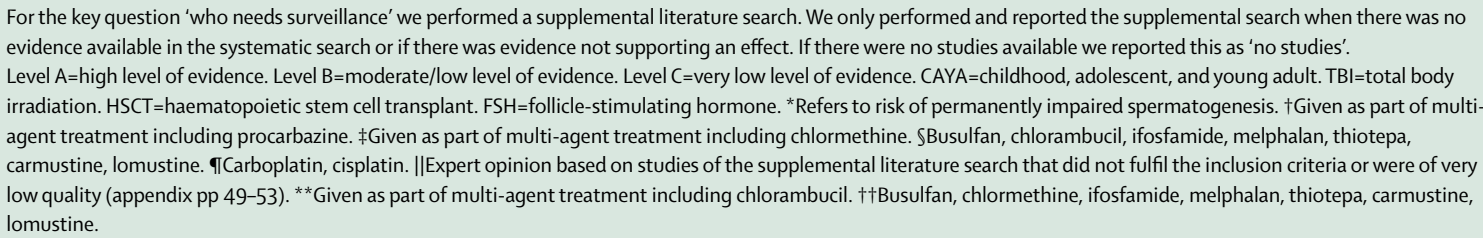 } \\
\hline
\end{tabular}

may be another important variable given evidence of variable fertility or even late recovery of spermatogenesis during prolonged follow-up in some survivors..$^{45-49}$ Even when these factors are controlled in studies whereby patients have received the same treatment exposures and have similar follow-up durations, interindividual differences in vulnerability persist, possibly reflecting genetic influences..$^{31,50}$

Several studies have suggested an association between alkylating agent dose and the probability of impaired spermatogenesis. ${ }^{31}$ Additionally, a 2016 study $^{51}$ found an association between higher cyclophosphamide, ifosfamide, procarbazine, and cisplatin doses and reduced probability of siring a pregnancy. However, although uncommon, azoospemia and oligozoospermia can occur after cyclophosphamide equivalent doses of less than $4000 \mathrm{mg} / \mathrm{m}^{2},{ }^{31}$ and it has not been possible to establish a completely safe lower dose threshold. ${ }^{5,50}$ Although the supplemental study suggested that testicular radiotherapy doses of more than 2-3 Gy are probably associated with a substantially increased risk of chronic spermatogeniesis impairment, whereas lower doses are unlikely to be associated with an increased risk, it is well known that even very small doses invariably cause acute impairment and that recovery does not always occur. ${ }^{4,52}$ Given this variability and the difficulty of excluding toxicity even with small doses, we concluded that any dose of potentially gonadotoxic alkylating agents or testicular radiotherapy should be regarded as possibly increasing the risk of impaired spermatogenesis.

Although the systematic search did not reveal evidence that alkylating agents, or radiotherapy potentially exposing the testes, are associated with an increased risk of testosterone deficiency, the supplemental search identified several studies suggesting that testicular radiotherapy probably leads to increased risk. This effect was observed in all studies describing testicular radiotherapy doses of 21-24 Gy or more and most studies evaluating doses of 


\section{Review}

\section{Impaired spermatogenesis}

General recommendation

Who needs surveillance?

Which surveillance modality should be used?

At what frequency and for how lon should surveillance be performed?

When should survivors with impaired spermatogenesis be referred?

\section{Testosterone deficiency}

\section{General recommendation}

Who needs surveillance?

\section{Which surveillance modality should be} used for prepubertal and peripubertal survivors? At what frequency and for how long?

Which surveillance modality should be used for postpubertal survivors? At what frequency and for how long?

When should survivors with abnormalities of pubertal development be referred?

\section{When should postpubertal survivors} with suspected testosterone deficiency be referred??

Physical sexual dysfunction

General recommendation

Who needs surveillance?

Which surveillance modality should be used?

When should survivors with suspected physical sexual dysfunction be referred?
Survivors treated with one or more potentially gonadotoxic agents, and their health-care providers, should be aware of the risk of impaired spermatogenesis and its implications for future fertility (level C evidence and supplemental literature search and expert opinion)

Counselling regarding the risk of impaired spermatogenesis and its implications for future fertility is recommended for survivors treated with: - Cyclophosphamide, chlormethine, procarbazine (level C evidence), busulfan and cyclophosphamide or fludarabine and melphalan for haematopoietic stem-cell transplant, ifosfamide (supplemental literature search and expert opinion)

- Radiotherapy potentially exposing testes (supplemental literature search and expert opinion)

In survivors who desire assessment about possible future fertility after treatment with potentially gonadotoxic chemotherapy or radiotherapy potentially exposing the testes, $\uparrow$ semen analysis is recommended as the gold standard primary surveillance modality for evaluation of spermatogenesis (expert opinion)

Clinical measurement of testicular volume and of follicle-stimulating hormone and inhibin B might be reasonable for the identification of impaired spermatogenesis in survivors treated with potentially gonadotoxic chemotherapy or radiotherapy potentially exposing the testes* in whom semen analysis has been declined or is not possible and who desire assessment about possible future fertility. Be aware of the diagnostic limitations of these tests that may result in false positives or false negatives (level B evidence)

Surveillance for impaired spermatogenesis should be performed only at the request of the survivor after informed discussion or when paternity is desired in the foreseeable future (expert opinion)

Referral to a male reproductive health specialist should be offered to survivors with severely impaired spermatogenesis, defined as severe oligospermia (sperm counts $\leq 5 \times 10^{6} / \mathrm{mL}$ ), or to those who are seeking paternity after potentially gonadotoxic chemotherapy or radiotherapy potentially exposing the testes, and to those whose attempts to conceive have been unsuccessful for 6 months or more, regardless of sperm count, for detailed specialist counselling or consideration of sperm cryopreservation if not already performed (expert opinion)

Survivors treated with a potentially gonadotoxic agent, and their health-care providers, should be aware of the risk of testosterone deficiency and its implications for future health and fertility (supplemental literature search and expert opinion)

Counselling regarding the risk of testosterone deficiency and its implications for future health and fertility is recommended for survivors treated with radiotherapy potentially exposing the testes to $12 \mathrm{~Gy}$ or more or with total body irradiation (supplemental literature search and expert opinion)

Monitoring of growth (height) and pubertal development and progression (Tanner stage including testicular volume) $† \$ \$$ is recommended for prepubertal and peripubertal survivors treated with radiotherapy potentially exposing the testes to $12 \mathrm{~Gy}$ or more or with total body irradiation (expert opinion)

Measurement of testosterone concentration in an early morning blood sample at clinically appropriate intervals is reasonable in postpubertal survivors treated with radiotherapy potentially exposing the testes to $12 \mathrm{~Gy}$ or more or with TBI (expert opinion). In the presence of clinical signs of hypogonadism, or of previous low-normal or borderline testosterone concentrations, or if it is not possible to obtain an early morning blood sample, it is reasonable to measure luteinising hormone concentration in addition to testosterone (expert opinion)

Referral to a paediatric endocrinologist is recommended for any survivor who has no signs of puberty by 14 years of age or failure of pubertal progression 1 (expert opinion)

Referral to a specialist in male reproductive health, andrology, endocrinology or urology (according to local referral pathways) is recommended for postpubertal survivors treated with radiotherapy potentially exposing the testes to $12 \mathrm{~Gy}$ or more or with $\mathrm{TBI}$, and in whom laboratory results suggest testosterone deficiency (expert opinion)

Survivors treated with one or more treatment modalities with potential to cause physical sexual dysfunction, or those who are hypogonadal, and their health-care providers, should be aware of the risk of physical sexual dysfunction (including erectile and ejaculatory dysfunction) and its implications for future health and fertility (supplemental literature search and expert opinion)

\section{Counselling regarding the risk of physical sexual dysfunction (including erectile and ejaculatory dysfunction) and its implications for future health and} fertility is recommended for survivors (supplemental literature search and expert opinion):

- Treated with surgery to the spinal cord, sympathetic nerves, or pelvis

- Treated with radiotherapy potentially exposing testes or pelvis

- Individuals who are hypogonadal

Providers should take a relevant sexual history in survivors treated with surgery to the spinal cord, sympathetic nerves, or pelvis, or radiotherapy potentially exposing testes or pelvis, or those who are hypogonadal (expert opinion)

Referral to a specialist in male reproductive health, andrology, endocrinology, or urology (according to local referral pathways) is recommended for survivors treated with surgery to the spinal cord, sympathetic nerves, or pelvis, or radiotherapy potentially exposing testes or pelvis, or those who are hypogonadal and who have symptoms suggesting physical sexual dysfunction (expert opinion)

$\square$ Strong recommendation to do $\square$ Moderate recommendation to do $\square$ Weak recommendation to do 
12 Gy or more, including TBI. One study ${ }^{42}$ found that TBI was associated with a lower testosterone concentration compared with no TBI on univariable analysis, but not on multivariable analysis. However, the analyses in this study considered testosterone concentration as a continuous variable. Therefore, the findings of the supplemental search were considered to have greater relevance to this clinical question, allowing a conclusion to be made that testicular radiotherapy doses of $12 \mathrm{~Gy}$ or more are probably associated with an increased risk of testosterone deficiency.

The systematic search did not identify any studies that clarified which survivors are at risk of physical sexual dysfunction, but the supplemental search found relevant evidence predominantly from studies of survivors of adult cancer or other patient populations, but reflecting clinical experience in CAYA cancer survivors. A 2016 study $^{53}$ on erectile dysfunction in male childhood cancer survivors was published too late to be included in our systematic Review, although an abstract from the same group was included in our supplemental search. ${ }^{54}$ Both publications described an increased risk of erectile dysfunction in survivors treated with higher dose $(\geq 10 \mathrm{~Gy}$ ) testicular radiotherapy, or surgery to the spinal cord or nerves, pelvis or prostate, when compared with their siblings. These sources of alternative evidence supported the conclusion that surgery to the spinal cord, sympathetic nerves or pelvis, radiotherapy potentially exposing the testes or pelvis, or any other treatment that causes hypogonadism, are each probably associated with an increased risk of physical sexual dysfunction.

A clear clinical and scientific consensus that semen analysis represents the gold standard for evaluating spermatogenesis exists. ${ }^{55}$ However, some survivors might decline or be unable to provide semen for analysis, and single results can be misleading. ${ }^{55}$ Health-care providers should be aware that results indicating severely impaired spermatogenesis can cause considerable distress to survivors, who might require appropriate psychological support. The effect of these results can be ameliorated by referral to a male reproductive health specialist to provide additional and more detailed counselling for the survivor, including information about future reproductive health options. This information can often provide some comfort for survivors.

Figure 2: Harmonised recommendations for gonadotoxicity surveillance for male CAYA cancer survivors

*Treatments with evidence and expert opinion for causing impaired spermatogenesis include cyclophosphamide, chlormethine, procarbazine (level C evidence), busulfan and cyclophosphamide or fludarabine and melphalan for $\mathrm{HSCT}$, ifosfamide, or radiotherapy potentially exposing the testes (supplemental literature search and expert opinion). †At least annually, with increasing frequency as clinically indicated depending on growth and pubertal progress. $¥$ Regular growth and pubertal monitoring should be started by no later than 12 years (and no earlier than 10 years) of age. \$The pubertal increase in growth velocity may be impaired if growth hormone deficiency is also present in survivors who received cranial radiation. 9 The absence of initiation of puberty (Tanner stage 2 ) in boys aged 14 years or older or failure to progress in pubertal stage for 6 months or more.
Previously published long-term follow-up guidelines ${ }^{18-20}$ have recommended measurement of testicular volume and serum FSH as surveillance. The DCOG guideline also recommended inhibin B measurement, particularly in the absence of semen analysis. A study ${ }^{30}$ in adult survivors of childhood Hodgkin lymphoma reported that only inhibin B showed an independent correlation with sperm concentration. Our results confirm that these measurements have fair diagnostic value for the detection of impaired spermatogenesis. Although testicular volume was not included as a clinical question, the studies identified confirmed that it has good diagnostic value (AUCs of ROC curves 0.7-0.9) in detecting oligozoospermia ${ }^{38}$ or excluding azoospermia. ${ }^{32,37}$ Therefore, these measurements might be reasonable in survivors in whom semen analysis is not available and who desire assessment about future fertility potential. However, FSH and inhibin B have considerable limitations for surveillance because they can give false positive or false negative results. For example, one of the included studies ${ }^{35}$ found that $22 \%$ of survivors with azoospermia had a normal (ie, false negative) FSH result, whereas $26 \%$ of those without azoospermia had an abnormal (ie, false positive) result. Analysis of the inhibin B to FSH ratio did not improve this situation. ${ }^{35}$ Therefore, clinical treatment and personal factors also need to be considered in individual survivors when judging whether or not to use these tests for surveillance.

No evidence was identified to inform recommendations regarding the frequency of surveillance for impaired spermatogenesis. This emphasises the importance of only undertaking such surveillance after informed discussion with survivors who desire paternity in the foreseeable future. The detection of severe oligozoospermia in individuals without previously stored sperm might suggest that referral to a male reproductive health specialist is appropriate for consideration of sperm cryopreservation to maximise the prospect of success with future attempts at assisted reproduction..$^{56}$ Consideration of sperm cryopreservation might be particularly important for survivors who are about to begin testosterone therapy, which could impair spermatogenesis further..$^{57}$ The rapid pace of advances in the field of male reproductive health, for example the use of surgical testicular sperm extraction followed by intracytoplasmic sperm injection, ${ }^{58}$ highlights the value of referral to a male reproductive health specialist even in azoospermic CAYA cancer survivors.

The recommendation for the surveillance modality for testosterone deficiency in postpubertal survivors is focused primarily on serum testosterone measurement, whilst maximising flexibility of clinical application by recommending measurement of $\mathrm{LH}$ in certain defined situations. The 2014 publication ${ }^{59}$ of validated age-related normal values for total testosterone will facilitate early detection and appropriate referral of testosterone deficiency, hence enabling reduction of morbidity. ${ }^{60}$ 
Panel: Gaps in knowledge and future directions for research

\section{Impaired spermatogenesis or testosterone deficiency}

- Risks of, and dose thresholds for, impaired spermatogenesis or testosterone deficiency in survivors treated with:

- Busulfan, chlorambucil, cyclophosphamide, ifosfamide, chlormethine, melphalan, and thiotepa (classical bifunctional alkylating agents)

- Dacarbazine, procarbazine, temozolomide (triazenes)

- Carboplatin, cisplatin (platinum agents)

- Carmustine, lomustine (nitrosoureas)

- Radiotherapy exposing the testes, including those treated with total body irradiation

(either alone or in combination)

- Influence of cyclophosphamide equivalent dose (calculated from formula that incorporates cumulative doses of busulfan, carmustine, chlorambucil, cyclophosphamide, ifosfamide, lomustine, chlormethine, melphalan, procarbazine, and thiotepa) on risk of impaired spermatogenesis and testosterone deficiency in survivors

- Potential recovery or deterioration of spermatogenesis or testosterone deficiency over time after these treatments

- Effect of age at treatment on the risk of impaired spermatogenesis or testosterone deficiency with any of the treatments listed above, risk of different cyclophosphamide equivalent doses, or potential recovery or deterioration

- Role of genetic susceptibility in thedevelopment of impaired spermatogenesis or testosterone deficiency in survivors treated with alkylating agents or radiotherapy exposing the testes

- Development of a risk prediction algorithm, taking into account demographic, treatment, and genetic variables, on risk of impaired spermatogenesis or testosterone deficiency in survivors

- Development of improved and more accessible biomarkers for impaired spermatogenesis or testosterone secretion

- Evaluation of potential psychological harm associated with excessive screening for impaired spermatogenesis, particularly from false positive results

- Diagnostic value of luteinising hormone to detect testosterone deficiency

- Evaluation of potential health benefits associated with appropriate identification of testosterone deficiency

Physical sexual dysfunction

- Risk of physical sexual dysfunction in male survivors treated with:

- Surgery to the spinal cord, sympathetic nerves, or pelvis

- Radiotherapy potentially exposing the testes, pelvis, or male reproductive organs

- Evaluation of potential benefits associated with appropriate identification of physical sexual dysfunction in male survivors

- Potential recovery or deterioration of physical sexual dysfunction in male survivors during lifespan because, although testosterone replacement treatment may be appropriate in deficient patients following accepted endocrine guidelines, the potential benefits and risks of treatment need to be considered appropriately for each individual. ${ }^{40,61-65}$

Definitions of failure of pubertal progression are not standardised, but our pragmatic recommendation to seek specialist referral following no advancement in Tanner stage over a 6-month period is intended to avoid delayed referral in a population of survivors at high risk of pubertal failure and consequent potential impairment of growth, metabolic health, bone mineral accretion, and quality of life as a result of testosterone deficiency. ${ }^{64}$

Several factors can influence the decision to undertake surveillance investigations and subsequently to refer survivors for specialist opinion, including perceptions of the reliability and utility of surveillance investigations, complexities in their interpretation, effects of the condition itself, treatments that could potentially be available to manage the condition, and possible treatment toxicities.

The task of harmonising male gonadal dysfunction surveillance guidelines was strengthened by our evidence-based approach with standardised outcome definitions, transparent presentation of the quality of available evidence and the strength of recommendation, and the multidisciplinary approach necessary to achieve consensus. When evidence was scarce for CAYA cancer survivors, we carefully extrapolated information from other populations.

The process used in the development of this guideline has revealed several substantial knowledge gaps for clinical research that could improve surveillance of gonadotoxicity in male CAYA cancer survivors. Research to address these gaps should be approached in a systematic, comprehensive manner by sufficiently large single-institution studies, or national and international multicentre collaborative projects.

This male gonadotoxicity surveillance guideline, and the international harmonisation initiative that underpins it, aims to improve health outcomes by facilitating more consistent long-term follow-up care for current male CAYA cancer survivors and promote strategically planned ongoing research that will inform future guidelines updates.

\section{Contributors}

RS, RLM, LCK, MMH, LSC, UD, LBK, SNu, MMvdH-E, HMvS, and DMG contributed to the conception and design of the study. RS, RLM, LCK, MMH, LSC, EB, AB, AB-S, MCB, RC, UD, AG, HI, KJ, LBK, JJL, LM, SNe, SNu, CP, MS, MMvdH-E, HMvS, WHWB, and DMG contributed to the search strategy, data extraction, interpretation of the data, and formulation of the recommendations. RS, RLM, LCK, MMH, LSC and DMG drafted, and EB, AB, AB-S, MCB, RC, UD, AG, HI, KJ, LBK, JJL, LM, SNe, SNu, CP, MS, MMvdH-E, HMvS, and WHWB critically revised the report. All authors approved the final version.

\section{Declaration of interests}

RS, LCK, EB, and MCB receive support from the 7th Framework Programme of the EU, PanCareSurfUp (257505). RLM receives support from the Dutch Cancer Society, Amsterdam, Netherlands (UVA 20114938) and the 7th Framework Programme of the EU, PanCareSurfUp 
(257505). MMH receives support from the American Lebanese Syrian Associated Charities (ALSAC). UD receives support from the Cancer Aid DKH-108128 and the 7th Framework Programme of the EU (PanCareLIFE) 602030. AG received personal fees from Bayer Pharmaceuticals and Ferring Pharmaceuticals for lecturing and advisory board membership.

\section{Acknowledgments}

We thank Edith Leclercq from Cochrane Childhood Cancer who developed the systematic literature search. We thank the following experts of the International Late Effects of Childhood Cancer Guideline Harmonization Group and members of the PanCareSurfUp Consortium for their participation in the international guideline harmonization process: Saro Armenian, Smita Bhatia, Natascia di Iorgi, Eva Frey, Riccardo Haupt, Lars Hjorth, Claudia Kuehni, Wendy Landier, Gill Levitt, Gisela Michel, Kevin Oeffinger, and Helena van der Pal. We thank Kyle Orwig, Chuck A Sklar, Jason Schwartz, Ryan Bishop, Jaap den Hartogh, Anouk Nijenhuis, and Jeroen te Dorsthorst for critically appraising the recommendations and manuscript.

\section{References}

1 Gatta G, Zigon G, Capocaccia R, et al. Survival of European children and young adults with cancer diagnosed 1995-2002. Eur J Cancer 2009; 45: 992-1005.

2 Howlader N, Noone AM, Krapcho M, et al. SEER cancer statistics review, 1975-2013. Bethseda, MD: National Cancer Institute. http:// seer.cancer.gov/csr/1975_2013/ (accessed Jan 6, 2017).

3 Geenen MM, Cardous-Ubbink MC, Kremer LC, et al. Medical assessment of adverse health outcomes in long-term survivors of childhood cancer. JAMA 2007; 297: 2705-15.

4 Oeffinger KC, Mertens AC, Sklar CA, et al. Chronic health conditions in adult survivors of childhood cancer. $N$ Engl $J$ Med 2006; 355: 1572-82

5 Green DM, Kawashima T, Stovall M, et al. Fertility of male survivor of childhood cancer: a report from the childhood cancer survivor study. J Clin Oncol 2010; 28: 332-39.

6 Kenney LB, Cohen LE, Shnorhavorian M, et al. Male reproductive health after childhood, adolescent, and young adult cancers: a report from the Children's Oncology Group. J Clin Oncol 2012; 30: 3408-16.

7 Romerius P, Stahl O, Moell C, et al. High risk of azoospermia in men treated for childhood cancer. Int J Androl 2010; 34: 69-76.

8 Romerius P, Ståhl O, Moell C, et al. Hypogonadism risk in men treated for childhood cancer. J Clin Endocrinol Metab 2009; 94: 4180-86

9 Zebrack BJ, Casillas J, Nohr L, Adams H, Zeltzer LK. Fertility issues for young adult survivors of childhood cancer. Psychooncology 2004; 13: 689-99.

10 Zebrack BJ, Foley S, Wittmann D, Leonard M. Sexual functioning in young adult survivors of childhood cancer. Pyschooncology 2010; 19: $814-22$.

11 Basaria S. Male hypogonadism. Lancet 2014; 383: 1250-63.

12 Wylie K, Rees M, Hackett H, et al. Androgens, health and sexuality in women and men. Hum Fertil 2010; 13: 277-97.

13 Bober SL, Zhou ES, Chen B, Manley PE, Kenney LB, Recklitis CJ. Sexual function in childhood cancer survivors: a report from Project REACH. J Sex Med 2013; 10: 2084-93.

14 Institute of Medicine. Clinical practice guidelines we can trust. Washington, DC: The National Academies Press, 2011.

15 Bero LA, Grilli R, Grimshaw JM, Harvey E, Oxman AD, Thomson MA. Closing the gap between research and practice: an overview of systematic reviews of interventions to promote the implementation of research findings. The Cochrane Effective Practice and Organization of Care Review Group. BMJ 1998; 317: 465-68.

16 Grimshaw JM, Russell IT. Effect of clinical guidelines on medical practice: a systematic review of rigorous evaluations. Lancet 1993; 342: 1317-22.

17 Woolf SH, Grol R, Hutchinson A, Eccles M, Grimshaw J. Clinical guidelines: potential benefits, limitations, and harms of clinical guidelines. BMJ 1999; 318: 527-30.

18 Skinner R, Wallace WHB, Levitt GA. United Kingdom Children's Cancer Study Group Late Effects Group. Therapy based long term follow up. Practice statement. 2005. http://www.cclg.org.uk/write/ MediaUploads/Member\%20area/Treatment\%20guidelines/LTFU full.pdf (accessed May 9, 2016).
19 Children's Oncology Group. Long-term follow-up guidelines for survivors of childhood, adolescent, and young adult cancers. 2013. http://www.survivorshipguidelines.org/ (accessed May 9, 2016).

20 Scottish Intercollegiate Guidelines Network. Long term follow up of survivors of childhood cancer. A national clinical guideline. 2013. http://www.sign.ac.uk/pdf/sign132.pdf (accessed Jan 6, 2017).

21 Landier W, Bhatia S, Eshelman DA, et al. Development of risk-based guidelines for pediatric cancer survivors: the Children's Oncology Group Long-Term Follow-Up Guidelines from the Children's Oncology Group Late Effects Committee and Nursing Discipline. J Clin Oncol 2004; 22: 4979-90.

22 Kremer LC, Mulder RL, Oeffinger KC, et al. A worldwide collaboration to harmonize guidelines for the long-term follow-up of childhood cancer survivors: a report from the International Late Effects of Childhood Cancer Guideline Harmonization Group. Pediatr Blood Cancer 2013; 60: 543-9.

23 Brown MC, Levitt GA, Frey E, et al. The views of European clinicians on guidelines for long-term follow-up of childhood cance survivors. Pediatr Blood Cancer 2015; 62: 322-28.

24 Tape TG. The area under an ROC curve. http://gim.unmc.edu/ dxtests/roc3.htm (accessed Jan 4, 2017).

25 Lee SJ, Schover LR, Partridge AH, et al. American Society of Clinical Oncology Recommendations on fertility preservation in cancer patients. J Clin Oncol 2006; 24: 2917-31.

26 van Waas M, Neggers SJCMM, te Winkel ML, Beishuizen A, Pieters R, van den Heuvel-Eibrink MM. Endocrine late sequelae in long-term survivors of childhood non-Hodgkin lymphoma. Ann Oncol 2012; 23: 1626-32.

27 Atkins D, Best D, Briss PA, et al, for the GRADE Working Group Grading quality of evidence and strength of recommendations. BMJ 2004; 328: 1490-94.

28 Gibbons RJ, Smith S, Antman E. American College of Cardiology/ American Heart Association clinical practice guidelines: part I: where do they come from? Circulation 2003; 107: 2979-86.

29 López Andreu JA, Fernández PJ, Ferris i Tortajada JF, et al. Persistent altered spermatogenesis in long-term childhood cancer survivors. Pediatr Hematol Oncol 2000; 16: 21-30.

30 van Beek RD, Smit M, van den Heuvel-Eibrink MM, et al. Inhibin B is superior to FSH as a serum marker for spermatogenesis in men treated for Hodgkin's lymphoma with chemotherapy during childhood. Hum Reprod 2007; 22: 3215-22.

31 Green DM, Liu W, Kutteh WH, et al. Cumulative alkylating agen exposure and semen parameters in adult survivors of childhood cancer: a report from the St Jude Lifetime Cohort Study. Lancet Oncol 2014; 15: 1215-23.

32 Wilhelmsson M, Vatanen A, Borgstrom B, et al. Adult testicular volume predicts spermatogenetic recovery after allogeneic HSCT in childhood and adolescence. Pediatr Blood Cancer 2014; 61: 1094-100.

33 Marshall WA, Tanner JM. Variations in the pattern of pubertal changes in girls. Arch Dis Child 1969; 44: 291-303.

34 Marshall WA, Tanner JM. Variations in the pattern of pubertal changes in boys. Arch Dis Child 1970; 45: 13-23.

35 Green DM, Zhu L, Zhang N, et al. Lack of specificity of plasma concentrations of inhibin B and follicle-stimulating hormone for identification of azoospermic survivors of childhood cancer: a report from the St Jude Lifetime Cohort Study. J Clin Oncol 2013; 31: 1324-28.

36 Rendtorff R, Beyer M, Muller A, et al. Low inhibin B levels alone are not a reliable marker of dysfunctional spermatogenesis in childhood cancer survivors. Andrologia 2012; 44: 219-25.

37 Jahnukainen K, Heikkinen R, Henriksson M, Cooper TG, Puukko-Viertomies LR, Mäkitie O. Semen quality and fertility in adult long-term survivors of childhood acute lymphoblastic leukemia. Fertil Steril 2011; 96: 837-42.

38 Lähteenmäki PM, Arola M, Suominen J, Salmi TT, Andersson AM, Toppari J. Male reproductive health after childhood cancer. Acta Paediatr 2008; 97: 935-42.

39 National Health Service Choices. Fertility treatments if you can't get pregnant. 2016. http://www.nhs.uk/conditions/pregnancy-andbaby/pages/fertility-treatments.aspx (accessed June 14, 2016).

40 Bhasin S, Cunningham GR, Hayes FJ, et al. Testosterone therapy in men with androgen deficiency syndromes: an Endocrine Society Clinical Practice Guideline. J Clin Endocrinol Metab 2010; 95: 2536-59. 
41 Siimes MA, Lie SO, Andersen O, Marky I, Rautonen J, Hertz H. Prophylactic cranial irradiation increases the risk of testicular damage in adult males surviving ALL in childhood. Med Pediatr Oncol 1993; 21: 117-21.

42 Tromp K, Claessens JJM, Knijnenburg SL, et al. Reproductive status in adult male long-term survivors of childhood cancer. Human Reprod 2011; 26: 1775-83.

43 Mackie EJ, Radford M, Shalet SM. Gonadal function following chemotherapy for childhood Hodgkin's disease. Med Pediatr Oncol 1996; 27: 74-78.

44 Ash P. The influence of radiation on fertility in man. Br J Radiology 1980; 53: 271-78.

45 Bujan L, Walschaerts M, Moinard N, et al. Impact of chemotherapy and radiotherapy for testicular germ cell tumors on spermatogenesis an sperm DNA: a multicentre prospective study from the CECOS network. Fertil Steril 2013; 100: 673-80.

46 Drasga RE, Einhorn LH, Williams SD, Patel DN, Stevens EE. Fertility after chemotherapy for testicular cancer. J Clin Oncol 1989; 1: $179-83$.

47 Johnson DH, Ainsworth JD, Linde RB, Greco FA. Testicular function following combination chemotherapy with cis-platin, vinblastine, and bleomycin. Med Pediatr Oncol 1984; 12: 233-38.

48 van Dorp W, van der Geest IMM, Laven JSE, et al. Gonadal function recovery in very long-term male survivors of childhood cancer. Eur J Cancer 2013; 49: 1280-86.

49 Wasilewski-Masker K, Seidel KD, Leisenring W, et al. Male infertility in long-term survivors of pediatric cancer: a report from the childhood cancer survivor study. J Cancer Surviv 2014; 8: 437-47.

50 Romerius P, Giwercman A, Moell C, et al. Estrogen receptor alpha single nucleotide polymorphism modifies the risk of azoospermia in childhood cancer survivors. Pharmacogenet Genomics 2011; 21: 263-69.

51 Chow EJ, Stratton KL, Leisenring WM, et al. Pregnancy after chemotherapy in male and female survivors of childhood cancer treated between 1970 and 1999: a report from the Childhood Cancer Survivor Study cohort. Lancet Oncol 2016; 17: 567-76.

52 Rowley MJ, Leach DR, Warner GA, Heller CG. Effect of graded doses of ionizing radiation on the human testis. Radiat Res 1974; 59: 665-78.

53 Ritenour CW, Seidel KD, Leisenring W, et al. Erectile dysfunction in male survivors of childhood cancer-a report from the Childhood Cancer Survivor Study. J Sex Med 2016; 13: 945-54.
54 Ritenour CWM, Seidel KD, Leisenring W, et al. Sexual health in male childhood cancer survivors: a Report from the Childhood Cancer Survivor Study (CCSS). J Sex Med 2014; 11: 147 (abstr).

55 World Health Organization. WHO laboratory manual for the examination and processing of human semen. Geneva: World Health Organization, 2010.

56 Koscinski I, Wittemer C, Lefebvre-Khalil V, Marcelli F, Defossez A Rigot JM. Optimal management of extreme oligozoospermia by an appropriate cryopreservation programme. Human Reprod 2007; 22: 2679-84.

57 Qoubaitary A, Meriggiola C, Ng CM, et al. Pharmacokinetics of testosterone undecanoate injected alone or in combination with norethisterone enanthate in healthy men. J Androl 2006; 27: 853-67.

58 Hsiao W, Stahl PJ, Osterberg EC, et al. Successful treatment of postchemotherapy azoospermia with microsurgical testicular sperm extraction: the Weill Cornell experience. J Clin Oncol 2011; 29: 1607-11.

59 Kelsey TW, Li LQ, Mitchell RT, Whelan A, Anderson RA, Wallace WHB. A validated age-related normative model for male total testosterone shows increasing variance but no decline after age 40 years. PLoS One 2014; 9: e109346.

60 McCabe MJ, Bancalari RE, Dattani MT. Diagnosis and evaluation of hypogonadism. Pediatr Endocrinol Rev 2014; 11 (suppl 2): 214-29.

61 Dohle GR, Arver S, Bettocchi C, Jones TH, Kliesch S, Punab M. Guidelines on male hypogonadism. 2015. http://uroweb.org/wpcontent/uploads/EAU-Guidelines-Male-Hypogonadism-2015.pdf (accessed May 13, 2016).

62 Dwyer AD, Phan-Hug F, Hauschild M, Elowe-Gruau E, Pitteloud N. Hypogonadism in adolescence. Eur J Endocrinol 2015; 173: R15-24.

63 Morales A, Bebb RA, Manjoo P, et al. Diagnosis and management of testosterone deficiency syndrome in men: clinical practice guideline. CMAJ 2015; 187: 1369-77.

64 Seftel AD, Kathrins M, Niederberger C. Critical update of the 2010 Endocrine Society Clinical Practice Guidelines for Male Hypogonadism: a systematic analysis. Mayo Clin Proc 2015; 90: 1104-15.

65 Watson S, Fuqua JS, Lee PA. Treatment of hypogonadism in males. Pediatr Endocrinol Rev 2014; 11 (suppl 2): 230-39. 